Supporting Information for

\title{
Design Considerations for Nanowire Heterojunctions in Solar Energy Conversion/Storage
} Applications

Kevin Hagedorn, ${ }^{1}$ Colin Forgacs, ${ }^{2}$ Sean Collins, ${ }^{1}$ and Stephen Maldonado ${ }^{1,3 *}$

1) Department of Chemistry

2) Department of Physics

3) Program in Applied Physics

$930 \mathrm{~N}$ University

Ann Arbor, Michigan 48109-1055

* To whom correspondence should be addressed. Phone: 734-647-4750.

E-mail: smald@umich.edu Home Page: http://www.umich.edu/ mgroup/ 


\section{S1. Light Transmittance through Methanolic Electrolyte}

The optical transparency of methanol containing $1 \mathrm{M} \mathrm{LiClO}_{4}, 199.5 \mathrm{mM}$ dimethylferrocene, and $0.5 \mathrm{mM}$ dimethylferrocenium was measured using a Varian Carey $5000 \mathrm{UV}-\mathrm{V}$ is spectrometer. A UV-Vis spectrum was obtained using a $1-\mathrm{cm}$ pathlength quartz cuvette filled with this electrolyte. For photoelectrode measurements, a $2 \mathrm{~mm}$ solution pathlength was used. The solution concentration was decreased by a factor of 5 to adjust the measured transmittance data to match the photoelectrochemical experimental conditions according to Eq S1,

$$
-\log \left(\frac{P_{\text {meas }}}{P_{0}}\right)=a b c
$$

where $a$ is the material absorptivity, $b$ is the pathlength, and $c$ is the solution concentration.

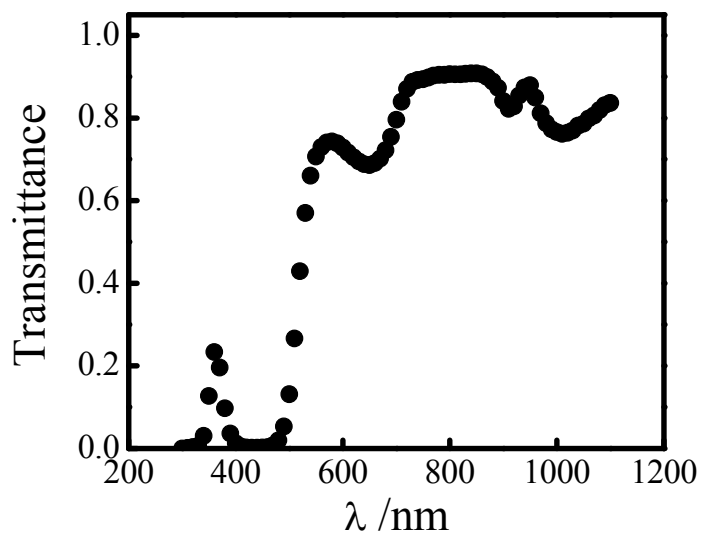

Figure S1. Approximate solution transmittance for a $2 \mathrm{~mm}$ beam pathlength through a methanolic electrolyte containing $199.5 \mathrm{mM}$ dimethylferrocene and $0.5 \mathrm{mM}$ dimethylferrocenium.

\section{S2. Determination of the Minority Carrier Diffusion Length}

Measurements of the external quantum yields for planar Si photoelectrodes in methanolic electrolyte containing dimethylferrocene/dimethylferrocenium were obtained at several wavelengths. Using the so-called Gärtner relation, these data were used to estimate $L_{\mathrm{p}}{ }^{1}$ 
External Quantum Yield = Internal Quantum Yield $\times(1-R)=\left(1-\frac{e^{-\alpha(\lambda) W}}{1+\alpha(\lambda) L_{p}}\right) \times(1-R)$

where $R$ is the reflectance of the $\mathrm{Si} /$ electrolyte interface, $\alpha(\lambda)$ is the wavelength-dependent absorptivity of $\mathrm{Si}$, and the rest of the terms have their same meanings as described in the main text. For wavelengths where $\alpha(\lambda)$ is much smaller relative to $W$, Eq S2 can be re-written as

$$
\frac{1}{\text { Internal Quantum Yield }}=1+\frac{1}{\alpha(\lambda)} \times \frac{1}{L_{p}}
$$
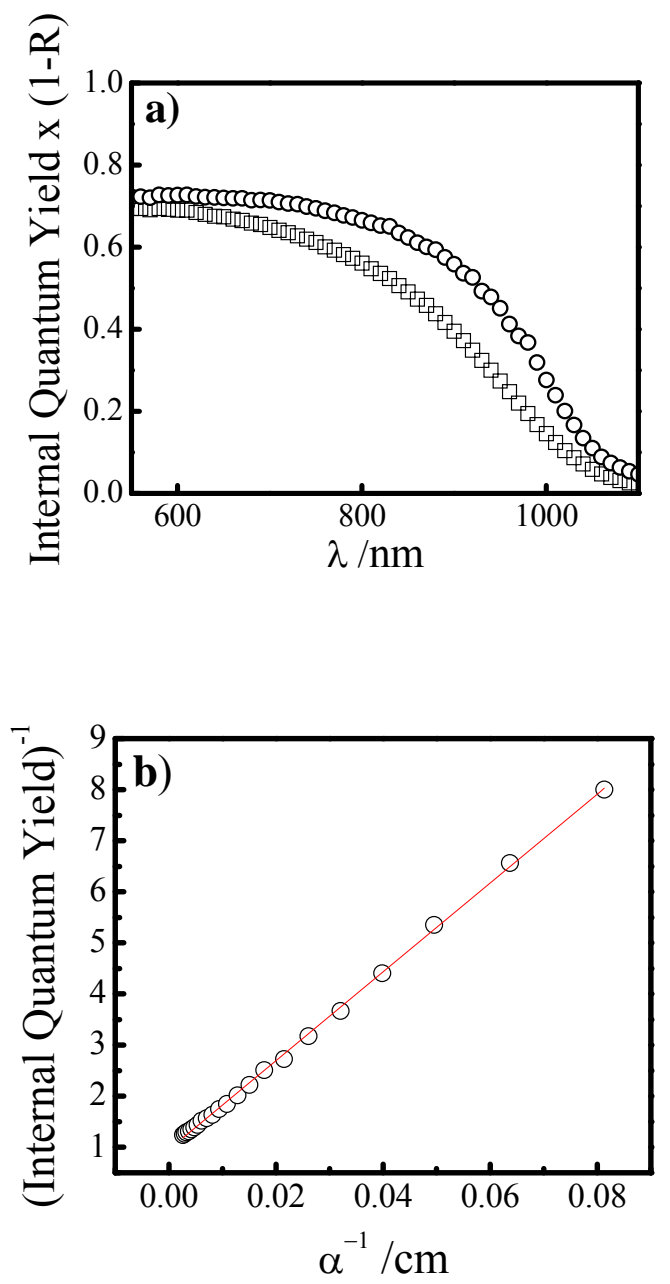


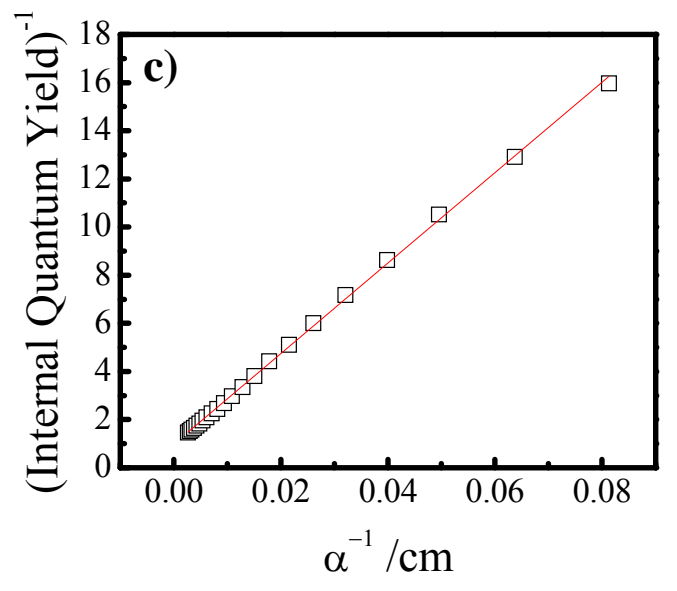

Figure S2. (a) Short-circuit external quantum yield (i.e. not corrected for reflectance losses) as a function of wavelength for the (open squares) heavily doped and (open circles) lightly doped planar Si photoelectrodes. These data are reproduced from Figure $4 \mathrm{a}$ in the main text. (b) Reciprocal short-circuit internal quantum yield values as a function of the wavelength-dependent optical penetration depth for the more lightly doped n-Si planar photoelectrodes. (c) Same as in (b) except these data are for the more heavily doped $\mathrm{n}$-Si planar photoelectrodes. The data in (b) and (c) were first corrected for ' $1-R$ ' losses using the known reflectivity of polished $\mathrm{Si}$ and the index of refraction of methanol. ${ }^{2}$

\section{S3. Reflectivity of Si Nanowire Film}

The total (specular + diffuse) reflectance at a Si nanowire film in contact with methanol was determined as described in the main text. These data were used to adjust the internal quantum efficiency values determined from numerical simulation with TeSCA to compare directly with the measured external quantum efficiency values. 


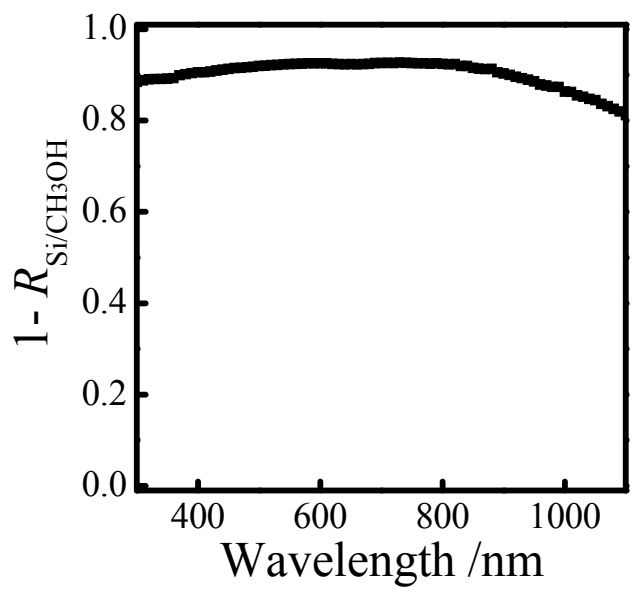

Figure S3. Total reflectance losses at the $\mathrm{n}-\mathrm{Si}$ nanowire $/ \mathrm{CH}_{3} \mathrm{OH}$ interface. The data represent the maximum values for the external quantum yield values measured at each wavelength.

\section{S4. TeSCA Simulations}

Recombination Processes A trap model ${ }^{3-4}$ was used in TeSCA to define the non-radiative bulk and surface recombination rates for the modeled semiconductor photoelectrode. For this recombination model, each trap state has an independent capture rate for electrons and holes that is defined as the product of the thermal velocity $\left(v_{\mathrm{i}}, \mathrm{cm} \mathrm{s}^{-1}\right)$ and the capture cross $\left(\sigma_{\mathrm{i}}, \mathrm{cm}^{2}\right)$ for each respective charge carrier,

$$
\begin{aligned}
& k_{n}=v_{n} \sigma_{n} \\
& k_{p}=v_{p} \sigma_{p}
\end{aligned}
$$

where $k_{\mathrm{n}}$ and $k_{\mathrm{p}}$ are the respective capture coefficients for electrons and holes at trap states in the bulk. Corresponding capture coefficients were calculated for electrons and holes at trap states at the electrode interface, respectively.

$$
\begin{aligned}
& k_{n, s}=v_{n} \sigma_{n} \\
& k_{p, s}=v_{p} \sigma_{p}
\end{aligned}
$$


Although this model could accommodate differences in capture processes for electrons and holes at traps, the results presented here assumed $v=v_{n}=v_{p}=10^{7} \mathrm{~cm} \mathrm{~s}^{-1}$ and $\sigma=\sigma_{n}=\sigma_{p}=10^{-15} \mathrm{~cm}^{2}$, in accord with experimentally observed values for crystalline $\mathrm{Si}^{5-6}$ The energy of the traps was set to be exactly in the middle of the bandgap. Explicit values for surface recombination rates, $S$, were then determined using Eq 3 in the main text. Explicit values for the minority carrier diffusion length were bound according to Eq S6,

$$
L_{p}=\sqrt{D_{p} \tau_{p}}=\sqrt{\frac{k_{B} T \mu_{p}}{q N_{t} k_{p}}}
$$

where $D_{\mathrm{p}}$ is the diffusion coefficient of minority carriers (holes), $\tau_{\mathrm{p}}$ is the lifetime of minority carriers, $N_{\mathrm{t}}$ is the bulk trap concentration, and the rest of the terms have their same meanings. To change the value of $L_{\mathrm{p}}$ for a given simulation, only the parameter $N_{\mathrm{t}}$ was adjusted. Auger recombination was also incorporated into the simulations, ${ }^{7}$ using recombination coefficients of $2 \times 10^{-31} \mathrm{~cm}^{6} \mathrm{~s}^{-1}$ for both electrons and holes. ${ }^{7}$

Heterogeneous Charge Transfer A Marcus-Gerischer formalism is used by TeSCA to model heterogeneous charge transfer at semiconductor/electrolyte interfaces, ${ }^{8}$

$$
\begin{aligned}
& j_{n}=j_{0, n}\left(\frac{n_{s}}{n_{0 s}}-1\right)=-q k_{e t} N_{C B}\left[d m F c^{+}\right] e^{\frac{-\left(q \Phi_{b}-\lambda_{r e}\right)^{2}}{4 k_{B} T \lambda_{r e}}}\left(\frac{n_{s}}{n_{0 s}}-1\right) \\
& j_{p}=j_{0, p}\left(\frac{p_{s}}{p_{0 s}}-1\right)=-q k_{h t} N_{V B}[d m F c] e^{\frac{-\left(\mathrm{E}_{g}-q \Phi_{b}-\lambda_{r e}\right)^{2}}{4 k_{B} T \lambda_{r e}}}\left(\frac{p_{s}}{p_{0 s}}-1\right)
\end{aligned}
$$

where $k_{e t}$ is the heterogeneous rate of electron transfer from the conduction band edge at optimal exoergicity, $k_{h t}$ is the heterogeneous rate of hole transfer from the valence band edge at optimal exoergicity, $[d m F c]$ is the concentration of dimethylferrocene, $\left[d m F c^{+}\right]$is the concentration of dimethylferrocenium, $\lambda_{r e}$ is the reorganization energy for the $\mathrm{dmFc} / \mathrm{dmFc}^{+}$redox couple, $n_{\mathrm{s}}$ is the surface concentration of electrons, $n_{0 \mathrm{~s}}$ is the surface concentration of electrons in the dark at equilibrium, $p_{\mathrm{s}}$ is the surface concentration of holes, and $p_{0 \mathrm{~s}}$ is the surface concentration of holes in the dark at equilibrium. A 
value of $0.5 \mathrm{eV}$ was used for $\lambda_{r e}$ based on the reorganization energies for fast outer-sphere redox couples. ${ }^{9}$ Precise measurements of $k_{e t}$ and $k_{h t}$ for $\mathrm{Si}$ in this system are not feasible since n-type and p-type $\mathrm{Si}$ electrodes are in strong inversion and accumulation conditions, respectively, when in contact with this electrolyte. Estimates for the magnitude of these standard rate constants were instead based on the following relations,

$$
\begin{aligned}
& k_{s c, e t}^{\mathrm{o}}=k_{e t} N_{C B} \\
& k_{s c, h t}^{o}=k_{h t} N_{V B} \\
& k_{s c, e t}^{\mathrm{o}} \leq k_{m}^{\mathrm{o}} \\
& k_{s c, h t}^{\mathrm{o}} \leq k_{m}^{\mathrm{o}}
\end{aligned}
$$

where $k_{s c}^{\mathrm{o}}$ is the heterogeneous charge-transfer standard rate constant at a semiconductor electrode ${ }^{10}$ and $k_{m}^{\mathrm{o}}$ is the standard rate constant for heterogeneous charge-transfer of the same redox couple at a metal electrode. Recent experimental measurements of $k_{m}^{o}$ for related ferrocene/ferrocenium redox couples in both aqueous and acetonitrile solutions using $\mathrm{Au}$ and $\mathrm{Pt}$ ultra-microelectrodes yielded values ranging between of 6.8 to $8.4 \mathrm{~cm} \mathrm{~s}^{-1}{ }^{11-12}$ These measurements imply values no greater than $2.9 \times 10^{-19}$ and $7.7 \mathrm{x}$ $10^{-19} \mathrm{~cm}^{4} \mathrm{~s}^{-1}$ for $k_{\mathrm{et}}$ and $k_{\mathrm{ht}}$ at Si electrodes, respectively. A conservative estimate of $1 \times 10^{-19} \mathrm{~cm}^{4} \mathrm{~s}^{-1}$ was used for both $k_{\text {et }}$ and $k_{\mathrm{ht}}$ for the presented simulations. Although TeSCA also has the capacity to include heterogeneous charge-transfer from surface states to acceptors/donors in solution, the rates for these processes were set to zero for the presented simulations. 
Table S1. TeSCA Simulation Parameters

\begin{tabular}{|c|c|c|c|}
\hline Parameter & Description & Value & Units \\
\hline$q \Phi_{\mathrm{b}}$ & barrier height & 1.0 & $\mathrm{eV}$ \\
\hline $\mathbf{E}_{\mathrm{g}}$ & bandgap & 1.12 & $\mathrm{eV}$ \\
\hline$n_{\mathrm{i}}$ & intrinsic carrier concentration & $1.45 \times 10^{10}$ & $\mathrm{~cm}^{-3}$ \\
\hline$\varepsilon_{\mathrm{Si}}$ & Si dielectric constant & 11.9 & \\
\hline$\varepsilon_{\text {electrolyte }}$ & electrolyte dielectric constant & 6 & \\
\hline$T$ & temperature & 300 & $\mathrm{~K}$ \\
\hline$N_{\mathrm{CB}}$ & effective density of states in conduction band & $2.8 \times 10^{19}$ & $\mathrm{~cm}^{-3}$ \\
\hline$N_{\mathrm{VB}}$ & effective density of states in valence band & $1.04 \times 10^{19}$ & $\mathrm{~cm}^{-3}$ \\
\hline$N_{\mathrm{D}}$ & dopant density & $10^{13}-10^{18}$ & $\mathrm{~cm}^{-3}$ \\
\hline$k_{\mathrm{n}}$ & bulk trap capture coefficient for electrons & $1 \times 10^{-8}$ & $\mathrm{~cm}^{3} \mathrm{~s}^{-1}$ \\
\hline$k_{\mathrm{p}}$ & bulk trap capture coefficient for holes & $1 \times 10^{-8}$ & $\mathrm{~cm}^{3} \mathrm{~s}^{-1}$ \\
\hline$k_{\mathrm{n}, \mathrm{s}}$ & surface trap capture coefficient for electrons & $1 \times 10^{-8}$ & $\mathrm{~cm}^{3} \mathrm{~s}^{-1}$ \\
\hline$k_{\mathrm{p}, \mathrm{s}}$ & surface trap capture coefficient for holes & $1 \times 10^{-8}$ & $\mathrm{~cm}^{3} \mathrm{~s}^{-1}$ \\
\hline$k_{\text {Aug,n }}$ & Auger recombination coefficient for electrons & $2 \times 10^{-31}$ & $\mathrm{~cm}^{6} \mathrm{~s}^{-1}$ \\
\hline$k_{\text {Aug,p }}$ & Auger recombination coefficient for holes & $2 \times 10^{-31}$ & $\mathrm{~cm}^{6} \mathrm{~s}^{-1}$ \\
\hline$N_{\mathrm{t}}$ & bulk trap density & $1 \times 10^{13}-5 \times 10^{13}$ & $\mathrm{~cm}^{-3}$ \\
\hline$N_{\mathrm{t}, \mathrm{s}}$ & surface trap density & $10^{8}-10^{9}$ & $\mathrm{~cm}^{-2}$ \\
\hline $\mathbf{E}_{\mathrm{s}}$ & energy of surface traps & $\mathbf{E}_{\mathrm{g}} / 2$ & $\mathrm{eV}$ \\
\hline$h$ & nanowire height & $15-100$ & um \\
\hline$r$ & nanowire radius & $25-500$ & $\mathrm{~nm}$ \\
\hline$\mu_{\mathrm{n}}$ & electron mobility & 1300 & $\mathrm{~cm}^{2} \mathrm{~V}^{-1} \mathrm{~s}^{-1}$ \\
\hline$\mu_{\mathrm{p}}$ & hole mobility & 450 & $\mathrm{~cm}^{2} \mathrm{~V}^{-1} \mathrm{~s}^{-1}$ \\
\hline$k_{\text {et }}$ & heterogeneous electron transfer rate constant & $10^{-19}$ & $\mathrm{~cm}^{4} \mathrm{~s}^{-1}$ \\
\hline$k_{\mathrm{ht}}$ & heterogeneous hole transfer rate constant & $10^{-19}$ & $\mathrm{~cm}^{4} \mathrm{~s}^{-1}$ \\
\hline$I_{0}$ & flux of photogenerated carriers & 0.02 & $\mathrm{C} \mathrm{cm}^{-2} \mathrm{~s}^{-1}$ \\
\hline$\alpha$ & absorptivity & $2.70-6390$ & $\mathrm{~cm}^{-1}$ \\
\hline$[\mathrm{dmFc}]$ & concentration of reduced redox couple & 0.0001 & $\mathrm{~mol} \mathrm{~cm}{ }^{-3}$ \\
\hline$\left[\mathrm{dmFc}^{+}\right]$ & concentration of oxidized redox couple & 0.0001 & $\mathrm{~mol} \mathrm{~cm}{ }^{-3}$ \\
\hline$\lambda_{r e}$ & reorganization energy & 0.5 & $\mathrm{eV}$ \\
\hline
\end{tabular}




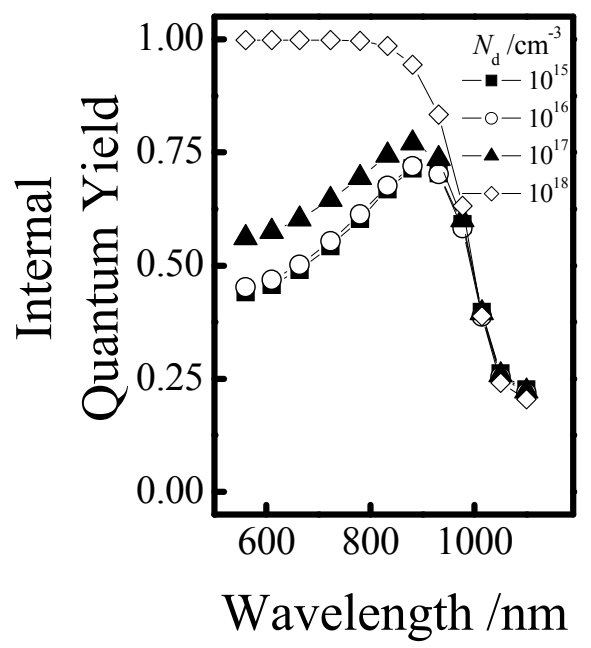

Figure S4. The internal quantum yield values predicted by TeSCA simulations for a $15 \mu \mathrm{m}$ tall $\mathrm{Si}$ nanowire film on top of a single-crystalline Si substrate. These data, in combination with the data in Figure S3, are shown as Figure $4 \mathrm{c}$ in the main text. 
Complete J-E photoresponses for Si nanowire photoelectrode under monochromatic illumination The following set of plots describe the full set of photoresponses discussed in the main text. For the following data sets, the data at $E=0 \mathrm{~V}$ were used to prepare Figure 5 in the main text.
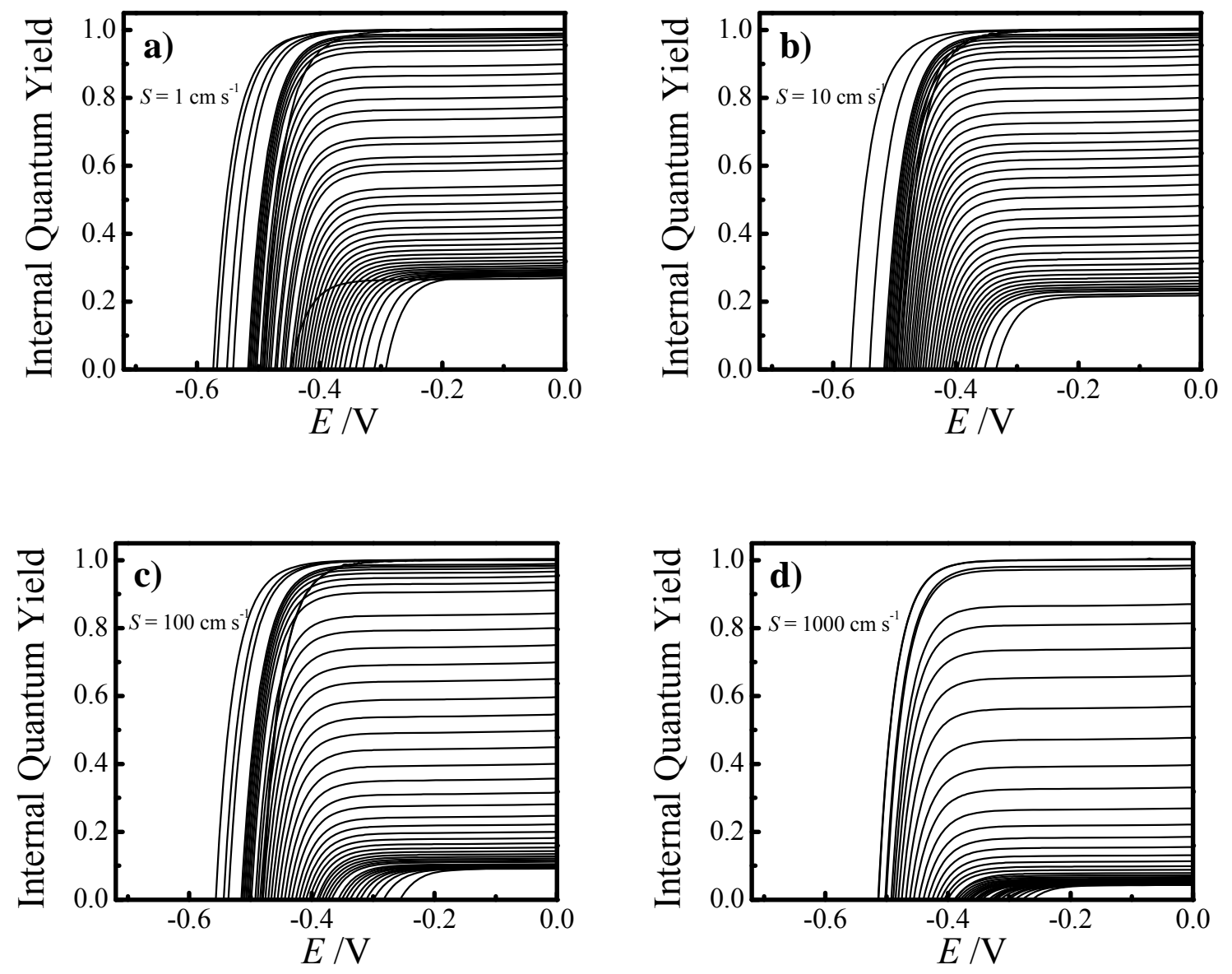

Figure S5. Simulated steady-state photocurrent-potential responses for a nanowire heterojunction with $r=50 \mathrm{~nm}$ wires, $q \Phi_{\mathrm{b}}=1 \mathrm{eV}$, and $L_{\mathrm{p}}=50 \mu \mathrm{m}$. The four sets of plots correspond to the data in Figure 5 a of the main text, with the value of $S$ indicated on each plot. 

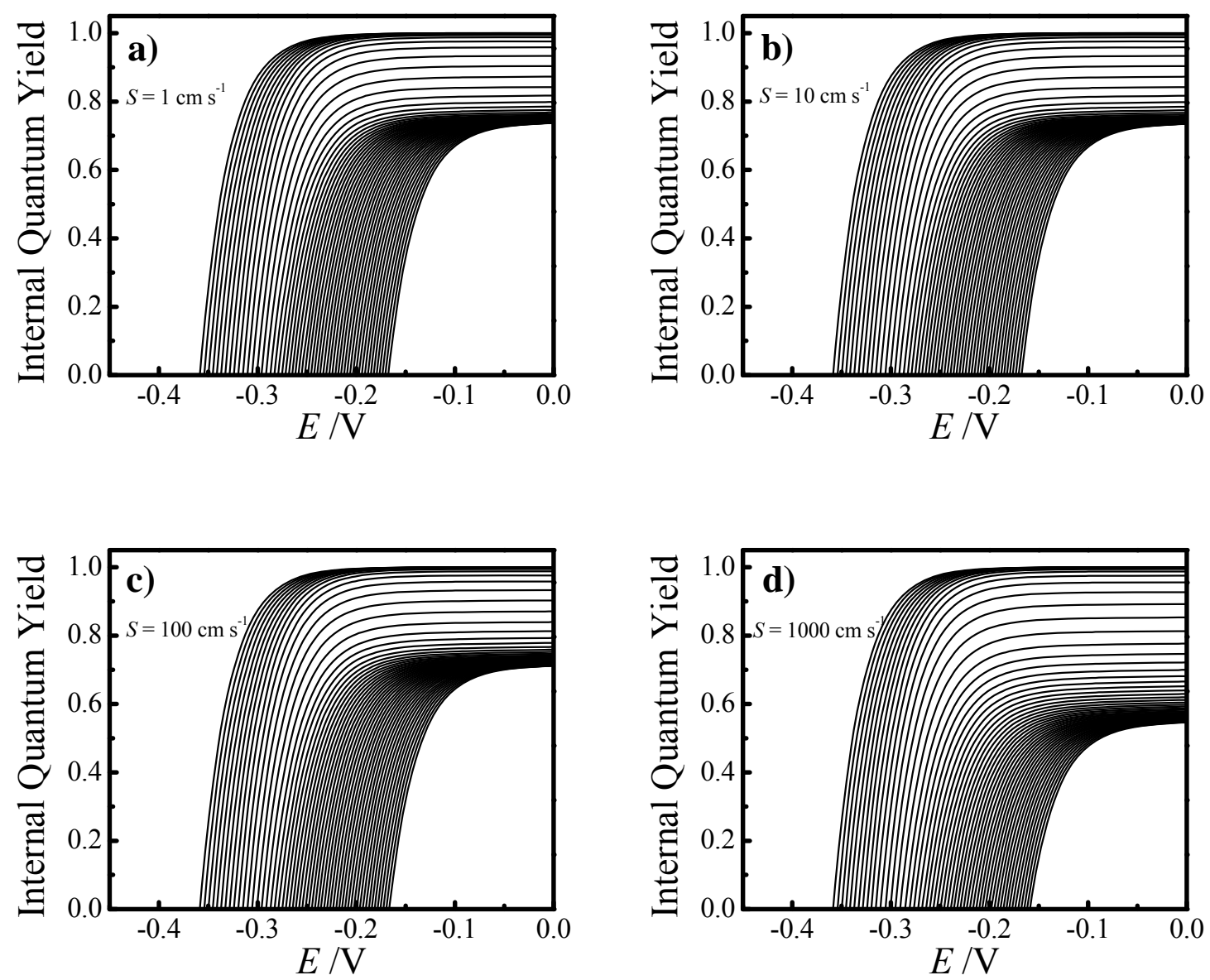

Figure S6. Simulated steady-state photocurrent-potential responses for a nanowire heterojunction with $r=500 \mathrm{~nm}$ wires, $q \Phi_{\mathrm{b}}=1 \mathrm{eV}$, and $L_{\mathrm{p}}=50 \mu \mathrm{m}$. The four sets of plots correspond to the data in Figure $5 \mathrm{~b}$ of the main text, with the value of $S$ indicated on each plot. 

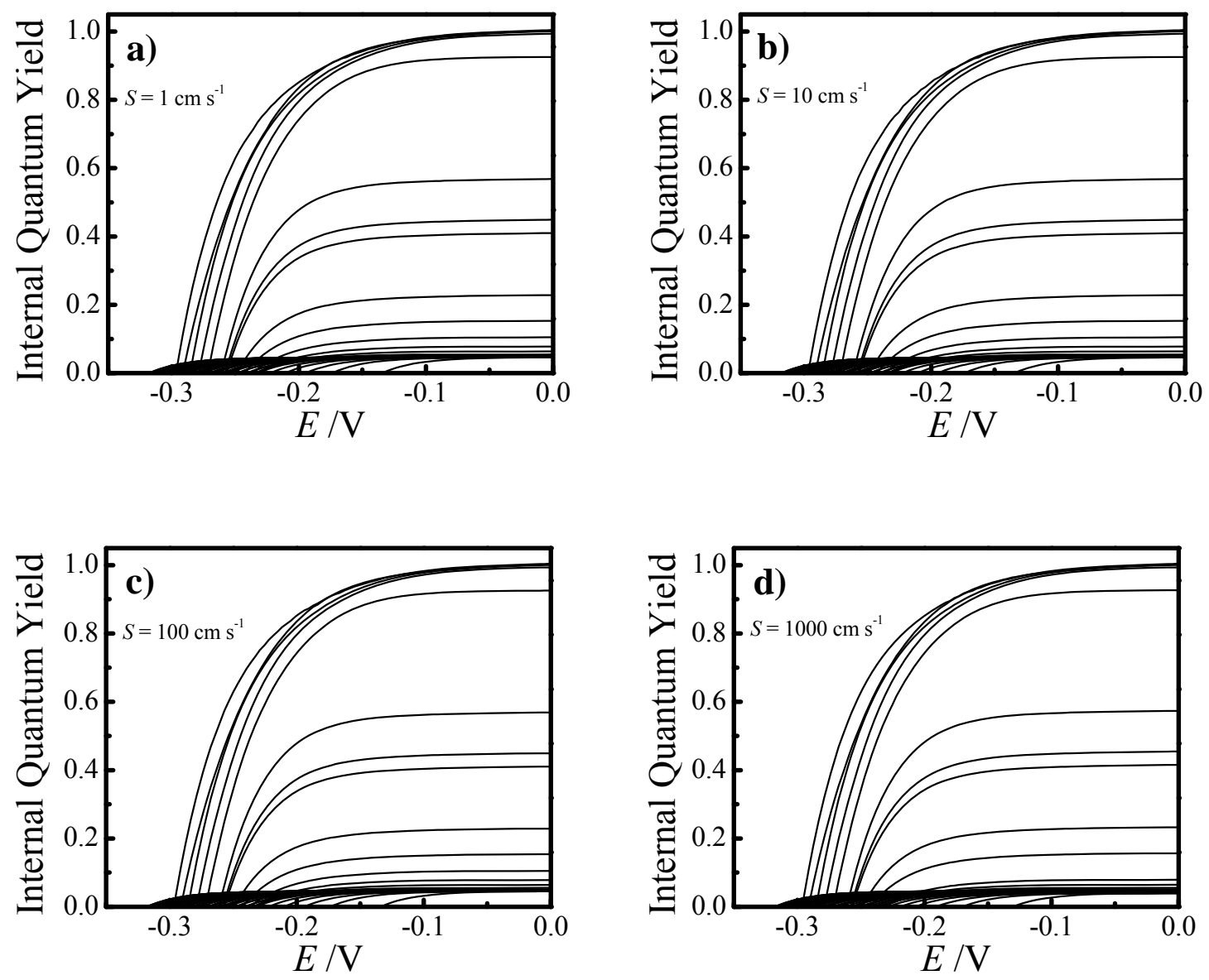

Figure S7. Simulated steady-state photocurrent-potential responses for a nanowire heterojunction with $r=50 \mathrm{~nm}$ wires, $q \Phi_{\mathrm{b}}=1 \mathrm{eV}$, and $L_{\mathrm{p}}=5 \mu \mathrm{m}$. The four sets of plots correspond to the data in Figure 5 c of the main text, with $S$ values indicated on each plot. 

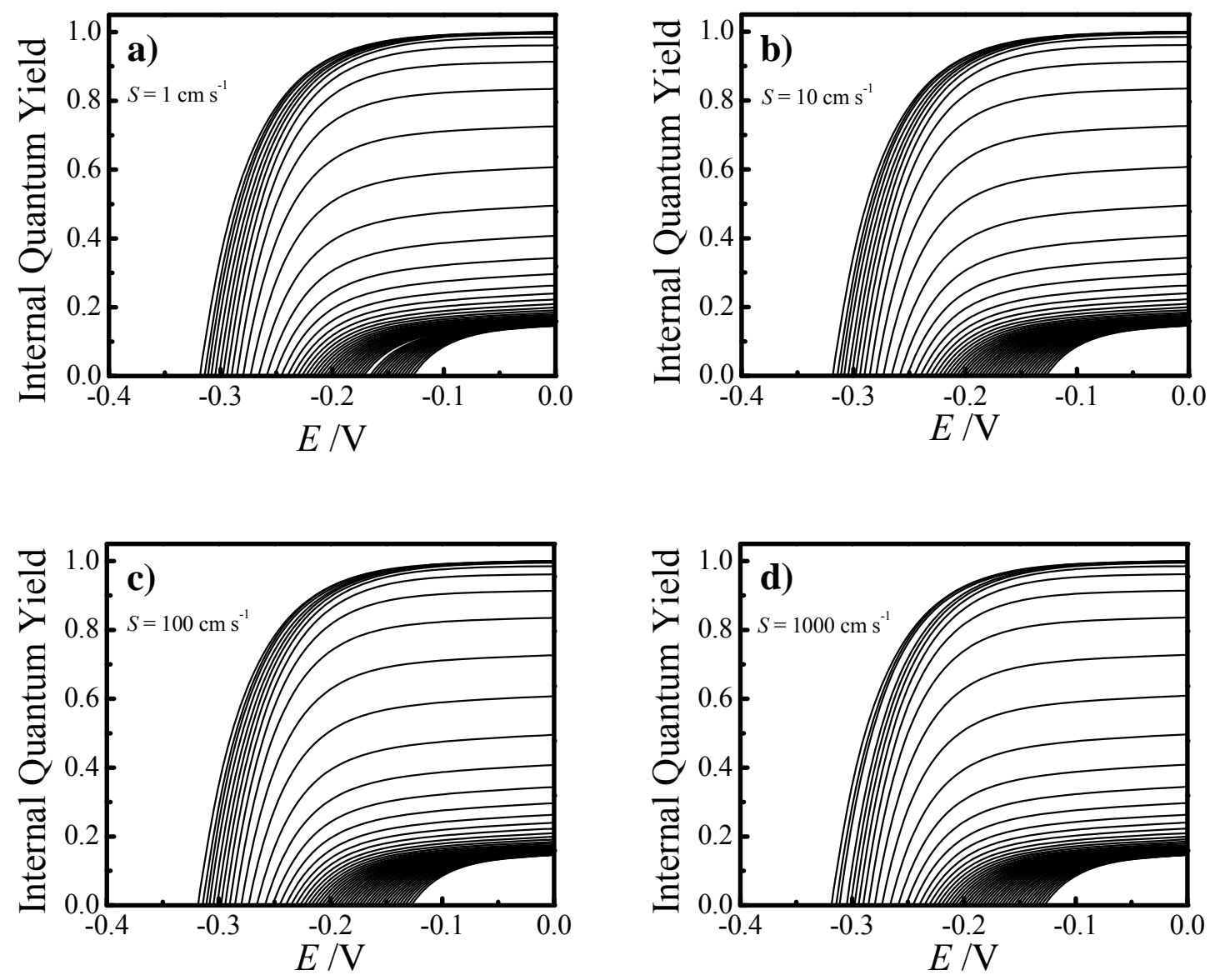

Figure S8. Simulated steady-state photocurrent-potential responses for a nanowire heterojunction with $r=500 \mathrm{~nm}$ wires, $q \Phi_{\mathrm{b}}=1 \mathrm{eV}$, and $L_{\mathrm{p}}=5 \mu \mathrm{m}$. The four sets of plots correspond to the data in Figure $5 \mathrm{~d}$ of the main text, with the value of $S$ indicated on each plot. 


\section{References.}

1. Gartner, W. W. Phys. Rev. 1959, 116, 84.

2. $\quad$ Green, M. A.; Keevers, M. Prog. Photovoltaics 1995, 3, 189.

3. $\quad$ Anz, S. J.; Kruger, O.; Lewis, N. S. J. Phys. Chem. B 1998, 102, 5625.

4. $\quad$ Anz, S. J.; Lewis, N. S. J. Phys. Chem. B 1999, 103, 3908.

5. Sze, S. M.; Ng, K. K. Physics of Semiconductor Devices; 3 ed.; John Wiley \& Sons, Inc.: Hoboken, 2007.

6. Wang, Y.; Cheung, K. P. Appl. Phys. Lett. 2007, 91, 113509(3).

7. Yablonovitch, E.; Allara, D. L.; Chang, C. C.; Gmitter, T.; Bright, T. B. Phys. Rev. Lett. 1986, 57, 249.

8. Gerischer, H. Adv. Electrochem. Electrochem. Engr. 1961, 1, 139.

9. Bard, A. J.; Faulkner, L. R. Electrochemical Methods: Fundamentals and Applications; John Wiley \& Sons, 2001.

10. Lewis, N. S. Ann. Rev. 1991, 42, 543.

11. Velmurugan, J.; Sun, P.; Mirkin, M. V. J. Phys. Chem. C 2009, 113, 459.

12. Sun, P.; Mirkin, M. V. Anal. Chem. 2006, 78, 6526. 